\title{
RINGS WITH A DERIVATION WHOSE IMAGE IS ZERO ON THE ASSOCIATORS
}

\author{
CHEN-TE YEN
}

\begin{abstract}
Let $R$ be a nonassociative ring, $N, M, L$ and $G$ the left nucleus,middle nucleus, right nucleus and nucleus respectively. Assume that $R$ is a ring with a derivation $d$ such that $d((R, R, R))=0$. It is shown that if $R$ is a simple ring then either $R$ is associative or $d(N \cap L)=0$; and if $R$ is a prime ring satisfying $R d(G) \subseteq N$ and $d(G) R \subseteq L$, or $d(G) R+R d(G) \subseteq M$ then either $R$ is associative or $d(G)=0$. These partially extend our previous results.
\end{abstract}

\section{Introduction}

Let $R$ be a nonassociative ring. We adopt the usual notations for associators and commutators: $(x, y, z)=(x y) z-x(y z)$ and $(x, y)=x y-y x$. We shall denote the left nucleus, middle nucleus, right nucleus and nucleus by $N, M, L$ and $G$ respectively. Thus $N, M, L$ and $G$ consists of all elements $n$ such that $(n, R, R)=0,(R, n, R)=0$, $(R, R, n)=0$ and $(n, R, R)=(R, n, R)=(R, R, n)=0$ respectively. An additive mapping $d$ on $R$ is called a derivation if $d(x y)=d(x) y+x d(y)$ for all $x, y$ in $R$. $R$ is called semiprime if the only ideal of $R$ which squares to zero is the zero ideal. $R$ is called prime if the product of any two nonzero ideals of $R$ is nonzero. $R$ is called simple if $R$ is the only nonzero ideal of $R$. Clearly, a prime ring is a semiprime ring. If $R$ is a simple ring, then $R^{2}=0$ or $R^{2}=R$; in the former case $R$ is commutative and associative. So, if $R$ is a simple ring then we assume that $R^{2}=R$. Thus a simple ring is a prime ring. Recently, Suh [1] proved that if $R$ is a prime ring with a derivation $d$ such that $d(R) \subseteq G$ then either $R$ is associative or $d^{3}=0$. In the notes [2]-[4], we improved and generalized this result. In this note, we prove that if $R$ is a simple ring with a derivation $d$ such that $d((R, R, R))=0$ then either $R$ is associative or $d(N \cap L)=0$. We also show that if

Received August 2, 1993.

1991 Mathematics Subject Classification. Primary 17A36.

Key words and phrases. Nonassociative ring, nucleus, derivation, Lie ideal, $d$-invariant, semiprime ring, prime ring, simple ring. 
$R$ is a prime ring with a derivation $d$ such that $d((R, R, R))=0$, and $R d(G) \subseteq N$ and $d(G) R \subseteq L$, or $d(G) R+R d(G) \subseteq M$, then either $R$ is associative or $d(G)=0$.

Let $R$ be a ring with a derivation $d$. Using the definition of $d$, and by a direct computation we have

$$
d((x, y, z))=(d(x), y, z)+(x, d(y), z)+(x, y, d(z)) \text { for all } x, y, z \text { in } \mathrm{R} .
$$

Assume that $d(R) \subseteq A$, where $A$ is a subring of $R$. For all $a \in A$ and $x \in R$, we obtain that $d(a x)=d(a) x+a d(x) \in A$ and $d(x a)=d(x) a+x d(a) \in A$. Since $a d(x) \in A$ and $d(x) a \in A$, these imply $d(a) x \in A$ and $x d(a) \in A$. Thus, $d(A) R+R d(A) \subseteq A$. On the other hand, if $d(R) \subseteq G$ then $d(G) R+R d(G) \subseteq G$ and by (1) we have $d((x, y, z))=0$ for all $x, y, z$ in $R$ and so $d((R, R, R))=0$. A nonempty subset $T$ of $R$ is called $d$-invariant if $d(T) \subseteq T$. An additive subgroup $S$ of $(R,+)$ is called a Lie ideal of $R$ if $(S, R) \subseteq S$.

\section{Results}

Let $R$ be a nonassociative ring. In every ring one may verify the Teichmüller identity

$$
(w x, y, z)-(w, x y, z)+(w, x, y z)=w(x, y, z)+(w, x, y) z \text { for all } w, x, y, z \text { in } R .
$$

Suppose that $n \in N$. Then with $w=n$ in (2) we obtain

$$
(n x, y, z)=n(x, y, z) \text { for all } n \text { in } N, \text { and all } x, y, z \text { in } R \text {. }
$$

Assume that $m \in L$. Then with $z=m$ in (2) we get

$$
(w, x, y m)=(w, x, y) m \text { for all } m \text { in } L, \text { and all } w, x, y \text { in } R \text {. }
$$

As consequences of (2), (3) and (4), we have that $N, M, L, N \cap M, M \cap L, N \cap L$ and $G$ are associative subrings of $R$. By (1), we see that all of these subrings are $d$-invariant.

Definition. The associator ideal $I$ of $R$ is the smallest ideal which contains all associators.

Note that $I$ may be characterized as all finite sums of associators and right (or left) multiples of associators, as a consequence of (2). Hence we have

$$
I=\sum(R, R, R)+(R, R, R) R=\sum(R, R, R)+R(R, R, R) .
$$

We assume that $R$ has a derivation $d$ which satisfies

$$
\text { (*) } \quad d((R, R, R))=0 .
$$

Using (2) and $(*)$, and by the definition of $d$, we get

$$
d(w)(x, y, z)+(w, x, y) d(z)=0 \text { for all } w, x, y, z \text { in } R .
$$


Suppose that $w \in N$. Then applying $d(w) \in N,(3)$ and (6), we have $(d(w) x, y, z)=$ $d(w)(x, y, z)=0$. Thus, $d(w) x \in N$ and $d(N)(R, R, R)=0$. Using $d(N) \subseteq N$, $d(N)(R, R, R)=0$ implies $d(N)((R, R, R) R)=0$. Combining these with (5) yields

$$
d(N) R \subseteq N \text { and } d(N) \cdot I=0 .
$$

By symmetry, using $d(L) \subseteq L,(4),(5)$ and (6), and as above we obtain

$$
R d(L) \subseteq L \text { and } I \cdot d(L)=0
$$

Theorem 1. If $R$ is a simple ring with a derivation d such that $d((R, R, R))=$ 0 , then either $R$ is associative or $d(N \cap L)=0$.

Proof. By the simplicity of $R$, we have that $I=0$ or $I=R$. If $I=0$, then $R$ is associative. Assume that $I=R$. Then by (7) and (8), we obtain $d(N) \cdot R=0$ and $R \cdot d(L)=0$. Hence, the ideal $B$ of $R$ generated by $d(N \cap L)$ is $B=\sum d(N \cap L)$. Thus, $B \cdot R=0$ implies $B=0$. So, $d(N \cap L)=0$, as desired.

Lemma 1. Let $R$ be a ring and $C$ a nonempty subset of $G$. Then $R C \subseteq N$ if and only if $C R \subseteq M$, and $R C \subseteq M$ if and only if $C R \subseteq L$.

Proof. Using $C \subseteq G$, and with $x \in C$ in (2) we get

$$
(w x, y, z)-(w, x y, z)=0 \text { for all } w, y, z \text { in } R \text {. }
$$

Thus, this implies $w x \in N$ if and only if $x y \in M$. Hence, $R C \subseteq N$ if and only if $C R \subseteq M$. The proof of the second assertion is similar.

Lemma 2 ([5], Lemma $\mathbb{1}$ ). Let $R$ be a ring and $C$ a nonempty subset of G. If $R C \subseteq N$ and $C R \subseteq L$, or $C R+R C \subseteq M$, then $C R+R C \subseteq M$, and the ideal $E$ of $R$ generated by $C$ is $E=\sum C+C R+R C+R \cdot C R$.

Proof. Obviously, $E$ is an additive subgroup of $(R,+)$. By the hypotheses and Lemma 1, we have that $C \subseteq G$ and $C R+R C \subseteq M$. Using these we obtain that $R C \cdot R=R \cdot C R$

$$
(R \cdot C R) R=R(C R \cdot R)=R\left(C \cdot R^{2}\right) \subseteq R \cdot C R
$$

and

$$
R(R \cdot C R)=R(R C \cdot R)=(R \cdot R C) R=\left(R^{2} \cdot C\right) R \subseteq(R \cdot C) R=R \cdot C R
$$

. At this point, we have verified that $E$ is an ideal of $R$.

Theorem 2. Let $R$ be a prime ring with a derivation d such that $d((R, R, R))$ $=0$. If $R d(G) \subseteq N$ and $d(G) R \subseteq L$, or $d(G) R+R d(G) \subseteq M$, then either $R$ is associative or $d(G)=0$. 
Proof. Applying $d(G) \subseteq G$ and Lemma 2, we see that the ideal $F$ of $R$ generated by $d(G)$ is

$$
F=\sum d(G)+d(G) R+R d(G)+R \cdot d(G) R
$$

and $d(G) R+R d(G) \subseteq M$.

Since $G \subseteq N$, by (7) we get $d(G) \cdot I=0$. Applying this, $d(G) \subseteq G$ and $d(G) R+$ $R d(G) \subseteq M$, we obtain $F \cdot I=0$. By the primeness of $R$, this implies either $F=0$ or $I=0$. Thus, either $d(G)=0$ or $R$ is associative, as desired.

Assume that $d(R) \subseteq G$. Then as in the Introduction, we obtain $d((R, R, R))=0$ and $d(G) R+R d(G) \subseteq G$. Hence, our results partially generalize the previous results. Applying the results above and Theorem 2, we have the following generalization of Suh's result.

Corollary 1 . If $R$ is a prime ring with a derivation $d$ such that $d(R) \subseteq G$, then either $R$ is associative or $d^{2}=0$.

In the course of the proof of Theorem 2, we obtain the

Corollary 2. Let $R$ be a semiprime ring with a derivation d such that $d((R, R, R))=0$ and $d(G) \subseteq I$. If $R d(G) \subseteq N$ and $d(G) R \subseteq L$, or $d(G) R+R d(G) \subseteq$ $M$, then $d(G)=0$.

Let $R$ be a ring with a derivation $d$ such that $d((R, R, R))=0$. If $N$ and $L$ are Lie ideals of $R$ then using these, (7) and (8), we have that $(R, d(N)) \subseteq(R, N) \subseteq N$ and $d(N) R \subseteq N$ imply $R d(G) \subseteq R d(N) \subseteq N$, and $(d(L), R) \subseteq(L, R) \subseteq L$ and $R d(L) \subseteq L$ imply $d(G) R \subseteq d(L) R \subseteq L$ respectively. Thus applying Theorem 2, we obtain

Corollary 3. Let $R$ be a prime ring with a derivation $d$ such that $d((R, R, R))$ $=0$. If $N$ and $L$ are Lie ideals of $R$, then either $R$ is associative or $d(G)=0$.

Corollary 4. Let $R$ be a semiprime ring with a derivation d such that $d((R, R, R))=0$ and $d(G) \subseteq I$. If $N$ and $L$ are Lie ideals of $R$, then $d(G)=0$.

\section{References}

[1] T. I. Suh, "Prime nonassociative rings with a special derivation," Abstracts of papers presented to the Amer. Math. Soc., 14(1993), 284.

[2] C. T. Yen, "Rings with a derivation whose image is contained in the nuclei," Tamkang J. Math., 25 (1994), 303-309.

[3] C. T. Yen, "Rings with a Jordan derivation whose image is contained in the nuclei or commutative center," submitted.

[4] C. T. Yen, "Prime ring with a derivation whose some power image is contained in the nucleus," submitted.

[5] C. T. Yen, "Nonassociative rings with a special derivation," submitted.

Department of Mathematics, Chung Yuan University, Chung Li, Taiwan, 320, Republic of China. 